\title{
Sensitivity of laser flare photometry compared to slit-lamp cell evaluation in monitoring anterior chamber inflammation in uveitis
}

\author{
Ottavio Bernasconi · Marina Papadia • \\ Carl P. Herbort
}

Received: 29 April 2010/ Accepted: 10 July 2010/Published online: 5 August 2010

(C) Springer Science+Business Media B.V. 2010

\begin{abstract}
To study the sensitivity of laser flare photometry (LFP) in monitoring anterior chamber inflammation by correlating LFP measurements with slit-lamp evaluation of aqueous cells in HLA-B27related uveitis in a prospective trial. Slit-lamp cell evaluation was correlated with LFP-measured flare in a masked fashion in HLA-B27-related uveitis patients receiving standard topical therapy. At the time of 50 and $90 \%$ LFP flare reduction, the corresponding reduction of cells was recorded and statistically
\end{abstract}

The results presented here apply only to slit-lamp models of flaremeters (Kowa FC-1000, Kowa FC-2000 and Kowa FM-500), but cannot be extrapolated to the FM-600 model which is ill-suited for use in uveitis.

O. Bernasconi · M. Papadia · C. P. Herbort ( $₫)$

Inflammatory and Retinal Eye Diseases,

Centre for Ophthalmic Specialised Care (COS),

Rue de la Grotte, 1003 Lausanne, Switzerland

e-mail: carl.herb@bluewin.ch

\section{O. Bernasconi}

Ospedale Regionale Bellinzona e Valli,

Bellinzona, Switzerland

\section{Papadia}

Department of Neurosciences, Ophthalmology and Genetics, University of Genova, Genoa, Italy

e-mail: marinapapadia@yahoo.com

C. P. Herbort

University of Lausanne, Lausanne, Switzerland compared using the sign test. Forty-three episodes (in 43 patients) of acute anterior HLA-B27-related uveitis were included. LFP flare reduction and slitlamp cell reduction were strongly correlated. LFP was significantly more sensitive for both $50 \%(P=$ $0.001)$ and $90 \%(P=0.02)$ LFP flare reduction in assessing the decrease of anterior chamber inflammation. LFP was superior to slit-lamp cell evaluation in monitoring anterior chamber inflammation in uveitis. Flare, becoming a quantitative parameter when measured by LFP, rather than cells, should be considered the gold standard to measure anterior chamber inflammation in uveitis.

Keywords Laser flare photometry .

Intraocular inflammation · Quantitative method

\section{Introduction}

In 1959, a standardization system for the evaluation of intra-ocular inflammatory activity in uveitis was established and has been almost universally used for more than 40 years [1, 2]. In 2004 a panel of uveitis specialists that convened to establish new universal criteria for the standardization of uveitis nomenclature (SUN) re-adopted this grading system essentially unchanged despite the fact that laser flare photometry (LFP), a precise new technology for the grading of intra-ocular inflammation had been available for 
more than 20 years [3]. Despite these efforts of standardization, the assessment of inflammation (aqueous cells and flare) still remains subjective with large intra-observer and inter-observer variations. Aqueous flare and cells are the most useful parameters to evaluate the progression or regression of inflammation in the anterior segment. Because anterior chamber cells could be quantified to a certain extent, this was considered by uveitis specialists to be the predominant parameter in anterior inflammation [4]. Flare was considered less useful because it could not be evaluated as accurately as cells. The slit-lamp assessment of both flare and cells in the aqueous humor is based on the same optical principle of recording back-scattered light particles (photons) from a light beam directed into the anterior chamber [5].

LFP, a new technology commercially available since 1989, is based on the same principle as slitlamp flare evaluation, measuring back-scattered light from protein particles in the anterior chamber [6]. It differs, however, from slit-lamp flare evaluation in several crucial aspects. The light source (incoming light) in LFP is a laser beam, by definition monochromatic, emitting a constant quantity of photons over time, whereas the incoming light in slit-lamp flare evaluation is polychromatic and subject to fluctuations. In slit-lamp flare evaluation the detector is the human eye and the data are analyzed by the human brain, whereas in LFP the detector is a photodetector/photomultiplier and the data are analyzed by a computer [6]. This represents a tremendous gain of sensitivity, making flare the only inflammatory parameter that can be quantified to date. The extent of sensitivity gain over the classic slit-lamp flare evaluation becomes evident when considering the two scales. A scale ranging from 1-4 in slit-lamp flare evaluation compared to a scale ranging from 4 photons/millisecond ( $\mathrm{ph} / \mathrm{ms}$, the normal flare value in a non-inflamed eye) to values as high as $1000 \mathrm{ph} / \mathrm{ms}$ in LFP indicates the difference of sensitivities [7]. Precise follow-up of inflammation is now possible not only for flare levels that are clinically apparent but also in low or subclinical flare states on one side and very high flare states on the other side; two situations where the human eye is absolutely unable to measure flare variations [8].

LFP has become the standard method in evaluating post-surgical anti-inflammatory therapy such as in post-cataract inflammation [9-11]. It has also been shown to be reliable in monitoring therapy and predicting inflammatory recurrences in posterior uveitis when a sufficient level of associated bloodaqueous barrier disruption (flare) is present [12].

In this prospective masked trial the purpose was to compare the sensitivity of LFP in detecting changes in inflammation level in comparison to aqueous cell determination by slit-lamp examination and thereby to establish the accuracy, the superiority as compared to slit-lamp cell analysis, and the reliability and consistency of LFP for the determination, grading and follow-up of inflammation in acute anterior HLA-B27-related uveitis

\section{Patients and methods}

Patients known to be HLA B27-positive who were seen for a recurrent episode of inflammation, who had not been on therapy and had been asymptomatic for more than two months, or patients who presented with a first episode of non-granulomatous anterior uveitis of sudden onset that tested positive for the HLA B27 antigen, seen in the uveitis clinic of Hôpital Jules Gonin and in the Centre for Ophthalmic Specialised Ophthalmic Care (COS), were included in the study.

The study was approved by the local IRB and the patient's consent was obtained according to the Declaration of Helsinki.

Patients were evaluated clinically and by LFP at presentation and on days D1, D2-4, D7, D14, D21, D28 and on months M2 and M6.

\section{Clinical evaluation}

The clinical evaluation was performed by a clinician who was not aware of the LFP measurements. Specific clinical items were evaluated. Symptoms including photophobia and pain were graded from 0 to 3. Best-corrected visual acuity (BCVA) was measured.

Slit-lamp examination comprised the evaluation of conjunctival hyperhemia, graded from 0 to 3. Flare was graded from 0treit slit-lamp with a slit-lamp beam $\pm 3 \mathrm{~mm}$ length and $1 \mathrm{~mm}$ width $(0=$ complete absence of flare; 1 = faint flare, barely detectable flare; 2 = moderate flare, iris and lens details 
clear; 3 = marked flare, iris and lens details hazy; $4=$ intense flare, fixed, coagulated aqueous humor with fibrin). Using the same beam parameters, aqueous cells were graded from to 4 ( $0=$ no cells; rare $=$ cells found with prolonged viewing; occasional $=1-4$ cells per field; $1=5-10$ cells per field; $2=11-20$ cells per field; $3=21-50$ cells per field; $4=>50$ cells per field).

The following additional signs were looked for: fibrin, keratic precipitates (KPs) (mutton fat KPs, when present, were an exclusion criterion), synechiae, graded from 0 to 4 , according to the number of quadrants involved and nodules (Koeppe and Busacca nodules, when present, were also an exclusion criterion).

Measurement of intra-ocular inflammation by LFP

Anterior chamber flare was measured by a masked investigator who was unaware of the clinical findings using a laser flare cell meter FC 1000 or the laser flare meter FM-500 (Kowa Co, Tokyo, Japan). The instrument consists of a $\mathrm{He}-\mathrm{Ne}$ or a diode laser, a photomultiplier and an analyzing unit. The laser beam, aimed into the anterior chamber, produces scattering of photons when proteins or particles are present [12-14]. The anterior chamber, isolated by the hemato-ocular barrier, is normally practically free of proteins and flare values measured with the LFP are very low, at a level of $4 \mathrm{ph} / \mathrm{ms}$. In the case of inflammation there is a rupture of this barrier with an influx of proteins and inflammatory cells which are measured in a determined window [13-15]. Every LFP examination included nine measurements. The two highest and the two lowest values were discarded giving a measurement average using five measurements. The LFP measurements were not available to the clinician and were recorded on a separate sheet.

\section{Therapeutical protocol}

At presentation the patients were dilated using phenylephrine $5 \%$, tropicamide $1 \%$ and scopolamine $0.25 \%$ drops in association with prednisolone acetate $1 \%$ drops (Pred Forte ${ }^{\circledR}$ ), one drop every $5 \mathrm{~min}$ (3 cycles). Thereafter, hourly prednisolone acetate 1\% drops were prescribed from 07:00 to 23:00 and prednisolone $0.5 \%$ ointment was used at night.
Dilatation was maintained using scopolamine $0.25 \%$ t.i.d. Tapering was based on AC cells following a precisely established treatment schedule. In the case of new fibrin formation after D0, occurrence of a hypopyon or occurrence of new synechiae, a periocular injection of bethamethasone (4\%) was given in addition to the topical treatment.

Statistical methods

For each patient, the time required to reach a specific reduction of the LFP value, namely a 50 or $90 \%$ gain, was recorded. In a simplified approach, we noted the first examination at which such a gain was observed. Because of the large scatter of inflammation levels, the non-parametric sign test was then used to establish whether the corresponding reduction of aqueous cells evaluated by slit-lamp examination was significantly higher or lower.

In a second approach by interpolation between examinations, the mean time to reach a 50 or $90 \%$ reduction for both LFP-evaluated flare and aqueous cell was estimated in order to provide a rough estimate of the time advantage of one measurement method over the other.

\section{Results}

Forty-three patients presenting with an episode of acute anterior HLA-B27-related uveitis were included. The mean age of the collective was $44 \pm 12$ years and included 30 male and 13 female patients.

The numerical values of the main parameters, LFP-evaluated flare, slit-lamp-evaluated flare and cells, visual acuity and IOP were recorded at presentation and on the follow-up visits and are shown in Table 1.

The evolution over time of LFP-measured flare values and of slit-lamp-evaluated aqueous cells are seen in Figs. 1 and 2. LFP flare reduction and slit-lamp cell reduction were strongly correlated $(P=0.9)$. The interpolated mean time for a $50 \%$ LFP flare reduction and aqueous cell reduction was 3.9 and 14 days, respectively. The $90 \%$ reduction time was 19.6 days for LFP flare and 40 days for slitlamp-evaluated aqueous cells showing a much higher sensitivity for LFP flare in assessing the evolution of the decrease of inflammation. 
Table 1 Mean LFP flare, slit-lamp flare and slit-lamp cells, best-corrected visual acuity (BCVA) and intra-ocular pressure (IOP) on the different measurement days

\begin{tabular}{lllllllll}
\hline & D0 & D1 & D2 - D4 & D7 & D14 & D21 & M2 & M6 \\
\hline LFP flare & $143 \pm 23.9$ & $101.8 \pm 29$ & $83.7 \pm 21.2$ & $51.7 \pm 13.7$ & $22.5 \pm 4.2$ & $15.7 \pm 2.5$ & $7.64 \pm 0.7$ & $5.03 \pm 0.33$ \\
SL flare & 2.28 & 2.0 & 1.42 & 1.07 & 0.77 & 0.58 & 0.16 & 0.014 \\
SL cells & 2.52 & 2.48 & 2.09 & 1.47 & 0.75 & 0.55 & 0.08 & 0 \\
BCVA & 0.9 & 0.77 & 0.87 & 0.92 & 0.97 & 1.05 & 1.13 & 1.2 \\
IOP & 12.2 & 11.9 & 13.7 & 13.0 & 13.0 & 12.8 & 14.8 & 14.1 \\
\hline
\end{tabular}

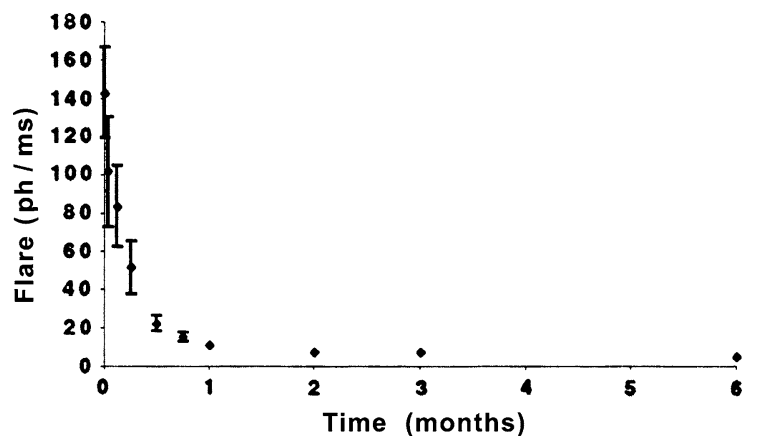

Fig. 1 Evolution of mean LFP flare \pm SD over time

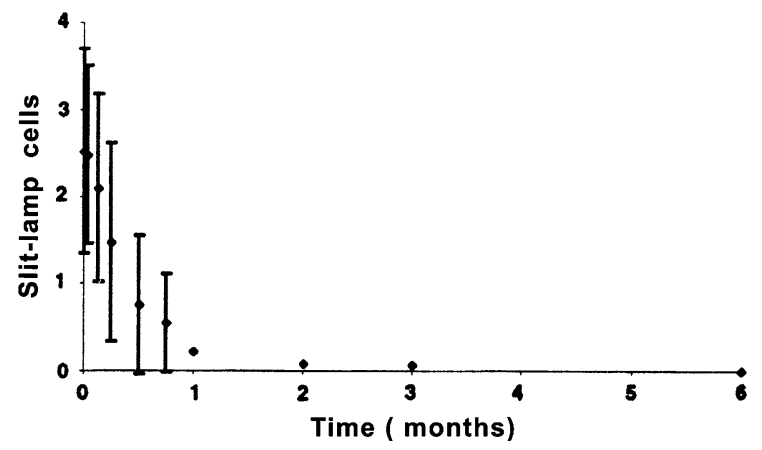

Fig. 2 Evolution of mean cell count \pm SD over time

The sensitivities of LFP and slit-lamp cell evaluation in assessing the reduction of inflammation are seen in Figs. 3 and 4. For both the 50\% and 90\% LFP flare reduction, LFP was significantly more sensitive than slit-lamp cells in assessing evolution of anterior chamber inflammation than slit-lamp evaluation of aqueous cells $(P<0.0001$ and $P<0.02)$. This means that for each patient reaching a 50\% and 90\% reduction of LFP flare the corresponding reduction of slit-lamp cells was noted, showing that LFP flare reduction was significantly more important than the reduction of cells.

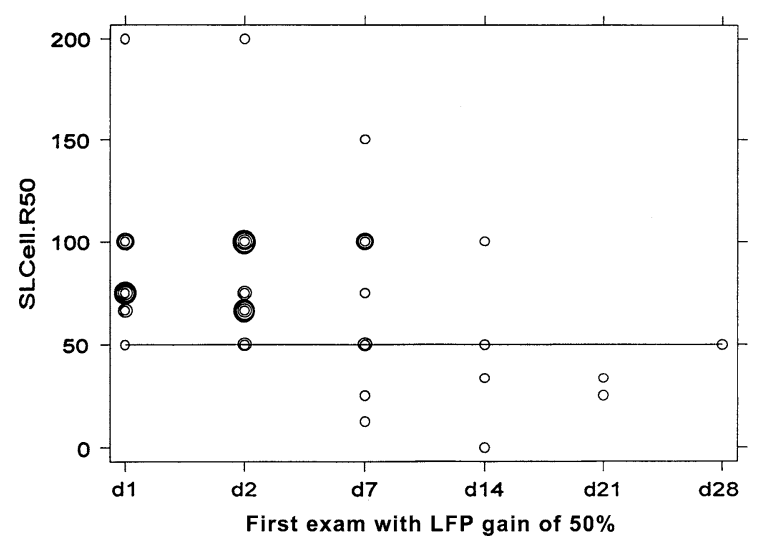

Fig. 3 Correlation between LFPflare reduction and slit-lamp cell reduction at 50\% LFP reduction time. Each circle represents one episode at 50\% LFP flare reduction. A great majority of cases have a $50 \%$ flare reduction on or before D7. At this moment there is not yet a $50 \%$ cell reduction in the majority of episodes (most circles are above the $50 \%$ cell reduction line)

\section{Discussion}

LFP has been shown to be superior to slit-lamp flare evaluation and to slit-lamp aqueous cell evaluation in traumatic (post-surgical, post-laser) inflammation and has been shown to be superior to slit-lamp flare evaluation in immunogenic (uveitic) inflammation $[7,9-12,16]$. In uveitis there is still a tendency to consider slit-lamp evaluation of aqueous cells as the gold standard for determining and monitoring anterior chamber inflammation. In this study our primary aim was to compare the accuracies of LFP-determined flare and slit-lamp evaluation of aqueous cells in assessing anterior chamber inflammation and to determine the superiority of this new quantitative method over classical slit-lamp cell evaluation of inflammation in uveitis patients. We therefore did not focus in the presentation of our results on the clinical 


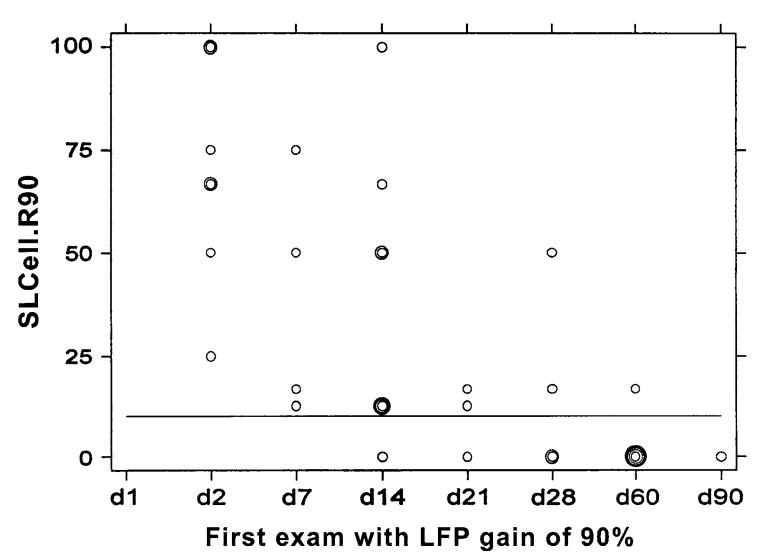

Fig. 4 Correlation between LFP flare reduction and slit-lamp cell reduction at $90 \%$ LFP reduction time. Each circle represents one episode with $90 \%$ LFP flare reduction. The great majority of cases have a $90 \%$ flare reduction on or before D14. At this moment there is not yet a $90 \%$ cell reduction in the majority of episodes (most circles are above the $90 \%$ cell reduction line). However, we can see that several episodes have a 90\% LFP flare reduction as late as D60 and these are all under the line of the corresponding $90 \%$ cell reduction, the consequence being that the statistical significance is lower $(P<0.02)$ than for the 50\% LFP flare reduction time-point $(P<0.0001)$. This means also that the blood-ocular barrier is still disrupted when cells have already disappeared

and laser flare photometric profile of acute anterior HLA-B27-related uveitis that was determined in previous studies $[16,17]$. The mean initial LFP flare and its evolution were comparable to our previous study, as were the final flare, mean duration of an episode and the clinical characteristics [16, 17]. Our data establish statistically for the first time that aqueous flare, when measured by LFP, is a more sensitive parameter than aqueous cells in assessing and following intra-ocular inflammation. The method is markedly more sensitive up to day seven of an episode when considering the $50 \%$ flare reduction and up to day 21 of an episode when considering the $90 \%$ flare reduction. The time advantage of LFP over slit-lamp cell evaluation is considerable as the mean time for a $50 \%$ reduction is 3.9 days for LFP flare and 14 days for cells. A $90 \%$ reduction is reached after 19.6 days for LFP flare and after 40 days for cells. We chose a very specific and well-determined type of uveitis to assess this new technology in uveitis. These results, however, can be extrapolated to any uveitisproducing inflammation in the anterior segment with the only exception of chronic blood-aqueous barrier disruption. Recently LFP flare profiles have been studied in panuveitis entities including Behçet's uveitis and Vogt-Koyanagi-Harada disease, showing persistence of slight elevation of LFP flare indicating persistent blood-ocular barrier disruption [18, 19]. This is the same in our study as shown by the graph of $90 \%$ flare reduction. While cells are resorbed, it takes time for the blood-ocular barrier to completely restore itself after severe inflammation, a fact that could not been found without LFP.

Although LFP is still able to measure additional new inflammation in these cases, the assessement of aqueous cells is still useful in monitoring intra-ocular inflammation. The certainty of having a quantitative, accurate and reliable method to assess even slight changes in intra-ocular inflammation will add security in the follow-up of inflammatory cases and should be considered the method of reference to measure intraocular inflammation. This is not only the case for acute inflammation such as HLA-B27-related uveitis but also in chronic inflammation such as uveitis related to idiopathic juvenile arthritis especially when there are no cells. We have shown that, even in the absence of cells, flare could be reduced with maximal therapy indicating that there can be active inflammation without the presence of cells [20]. LFP will not determine decision-making by itself but will contribute, together with clinical examination and other paraclinical tests such as fluorescein and indocyanine green angiography, when indicated, to a more accurate management of uveitis patients.

In conclusion, LFP was shown to be superior to slit-lamp cell evaluation in monitoring intra-ocular inflammation. By becoming a quantitative and objective parameter, when measured by LFP, flare rather than aqueous cells should be considered the reference parameter to monitor anterior chamber inflammation.

Acknowledgments This study was supported by a grant from Kowa Co., Electronic \& Optics Division Tokyo, Japan.

\section{References}

1. Hogan MJ, Kimura SJ, Thygeson P (1959) Signs and symptoms of uveitis. I. Anterior uveitis. Am J Ophthalmol 47(no. 5, pt. 2):155-170

2. Kimura SJ, Thygeson P, Hogan MJ (1959) Signs and symptoms of uveitis. II. Classification of the posterior manifestations of uveitis. Am J Ophthalmol 47 (no. 5, pt. 2): 171-176 
3. SUN Working group (2005) Standardization of uveitis nomenclature for reporting clinical data. Results of the first international workshop. Am J Ophthalmol 140:509-516

4. Kanski J (1987) Introduction. In: Kanski J (ed) Uveitis: a colour manual of diagnosis and treatment. Butterworths, London, pp 1-11

5. Spalton DJ (1993) Measurement of flare. Br J Ophthalmol 77:263-264

6. Sawa M, Tsurimaki Y, Tsuru T, Shimizu H (1988) New quantitative method to determine protein concentration and cell number in aqueous in vivo. Jpn $\mathrm{J}$ Ophthalmol 32:132-142

7. El-Maghraby A, Marzouki A, Matheen TM et al (1992) Reproducibility and validity of laser flare/cell meter measurements as an objective method of assessing intraocular inflammation. Arch Ophthalmol 110:960-962

8. Castella AP, Bercher L, Zografos L, Egger E, Herbort CP (1995) Study of blood-aqueous barrier in choroidal melanoma. Br J Ophthalmol 79:354-357

9. Mermoud A, Pittet N, Herbort CP (1992) Inflammation patterns after laser trabeculoplasty measured with the laser flare meter. Arch Ophthalmol 110:368-370

10. Herbort CP, Mermoud A, Schnyder C, Pittet N (1993) Anti-inflammatory effect of diclofenac drops after argon laser trabeculoplasty. Arch Ophthalmol 111:481-483

11. Herbort CP, Jauch A, Othenin-Girard P, Tritten JJ, Fsadni M (2000) Diclofenac drops to treat inflammation after cataract surgery. Acta Ophthalmol Scand 78:421-424

12. Guex-Crosier Y, Pittet N, Herbort CP (1995) Sensitivity of laser flare photometry to monitor inflammation in uveitis of the posterior segment. Ophthalmology 102:613-621
13. Sawa M (1990) Clinical application of laser flare-cell meter. Jpn J Ophthalmol 34:346-363

14. Shah SM, Spalton DJ, Taylor JC (1992) Correlations between laser flare measurements and anterior chamber protein concentrations. Invest Ophthalmol Vis Sci 33: 2878-2884

15. Shah SM, Spalton DJ, Smith SE (1991) Measurement of aqueous cells and flare in normal eyes. $\mathrm{Br} \mathrm{J}$ Ophthalmol 75:348-352

16. De Ancos E, Pittet N, Herbort CP (1994) Mesure quantitative de l'inflammation dans l'uvéite antérieure aiguë (UAA) HLA B27 à l'aide du laser flare-cell meter Kowa FC-1000. Klin Monatsbl Augenheilkd 204:330-333

17. Herbort CP, Guex-Crosier Y, de Ancos E, Pittet N (1997) Use of laser flare photometry (LFP) to assess and monitor inflammation in uveitis. Ophthalmology 104:64-72

18. Tugal-Tutkun I, Cingü K, Kir N, Yeniad B, Urgancioglu M, Gül A (2008) Use of laser flare-cell photometry to quantify intraocular inflammation in patients with Behçet's uveitis. Graefes Arch Clin Exp Ophthalmol 246:11691177

19. Fang W, Zhou H, Yang P, Huang X, Wang L, Kijlstra A (2008) Longitudinal quantification of aqueous flare and cells in Vogt-Koyanagi-Harada disease. Br J Ophthalmol 92:182-185

20. Herbort CP (2009) Laser flare photometry. In: Gupta A, Gupta V, Herbort CP, Khairallah M (eds) Uveitis: text and imaging. New Delhi, Jaypee, pp 28-49 